\title{
Riociguat for treatment of pulmonary hypertension in COPD: a translational study
}

\author{
Alexandra Pichl ${ }^{1,3}$, Natascha Sommer ${ }^{1,3}$, Mariola Bednorz ${ }^{1}$, Michael Seimetz ${ }^{1}$, \\ Stefan Hadzic ${ }^{1}$, Stefan Kuhnert ${ }^{1}$, Simone Kraut ${ }^{1}$, Elsa T. Roxlau ${ }^{1}$, \\ Baktybek Kojonazarov ${ }^{1}$, Jochen Wilhelm¹, Marija Gredic ${ }^{1}$, Henning Gall (1) ${ }^{1}$, \\ Khodr Tello', Manuel J. Richter ${ }^{1}$, Oleg Pak ${ }^{1}$, Aleksandar Petrovic ${ }^{1}$, \\ Matthias Hecker ${ }^{1}$, Ralph T. Schermuly ${ }^{1}$, Friedrich Grimminger ${ }^{1}$, \\ Werner Seeger (10 ${ }^{1,2}$, Hossein A. Ghofrani ${ }^{1}$ and Norbert Weissmann ${ }^{1}$
}

Affiliations: ${ }^{1}$ Excellence Cluster Cardiopulmonary System, University of Giessen and Marburg Lung Center (UGMLC), Member of the German Center for Lung Research (DZL), Justus Liebig University, Giessen, Germany. ${ }^{2}$ Max Planck Institute for Heart and Lung Research, Bad Nauheim, Germany. ${ }^{3}$ These two authors contributed equally to this work.

Correspondence: Norbert Weissmann, Excellence Cluster Cardiopulmonary System, University of Giessen and Marburg Lung Center (UGMLC), Aulweg 130, 35392 Giessen, Germany.

E-mail: norbert.weissmanndinnere.med.uni-giessen.de

@ERSpublications

The soluble guanylate cyclase stimulator riociguat reverses fully established cigarette smoke-induced pulmonary emphysema and hypertension in mice, and may have beneficial effects on $\mathrm{PH}$ in patients presenting with both PH and moderate COPD http://ow.ly/dGgL30oj7LC

Cite this article as: Pichl A, Sommer N, Bednorz M, et al. Riociguat for treatment of pulmonary hypertension in COPD: a translational study. Eur Respir J 2019; 53: 1802445 [https://doi.org/10.1183/ 13993003.02445-2018].

ABSTRACT Chronic obstructive pulmonary disease (COPD), which comprises the phenotypes of chronic bronchitis and emphysema, is often associated with pulmonary hypertension (PH). However, currently, no approved therapy exists for PH-COPD. Signalling of the nitric oxide (NO)-cyclic guanosine monophosphate (cGMP) axis plays an important role in $\mathrm{PH}$ and COPD.

We investigated the treatment effect of riociguat, which promotes the NO-cGMP pathway, in the mouse model of smoke-induced $\mathrm{PH}$ and emphysema in a curative approach, and retrospectively analysed the effect of riociguat treatment on $\mathrm{PH}$ in single patients with $\mathrm{PH}-\mathrm{COPD}$.

In mice with established $\mathrm{PH}$ and emphysema (after 8 months of cigarette smoke exposure), riociguat treatment for another 3 months fully reversed PH. Moreover, histological hallmarks of emphysema were decreased. Microarray analysis revealed involvement of different signalling pathways, e.g. related to matrix metalloproteinases (MMPs). MMP activity was decreased in vivo by riociguat. In PH-COPD patients treated with riociguat $(n=7)$, the pulmonary vascular resistance, airway resistance and circulating MMP levels decreased, while oxygenation at rest was not significantly changed.

Riociguat may be beneficial for treatment of PH-COPD. Further long-term prospective studies are necessary to investigate the tolerability, efficacy on functional parameters and effect specifically on pulmonary emphysema in COPD patients. 


\section{Introduction}

Chronic obstructive pulmonary disease (COPD) is one of the five major causes of death worldwide [1] and is largely caused by inhaled toxic agents, in Western countries mainly cigarette smoke. COPD is characterised by chronic bronchitis, emphysema and frequently pulmonary hypertension (PH). Underlying factors promoting $\mathrm{PH}$ in COPD (PH-COPD) are smoke-induced endothelial dysfunction, hypoxia and inflammation, enhanced in later stages by loss of vessels due to alveolar destruction [2]. PH-COPD is classified as group III of PH (PH due to lung diseases and/or hypoxia) according to the European Society of Cardiology/European Respiratory Society classification [3]. Although $\mathrm{PH}$ defined by mean pulmonary arterial pressure (mPAP) $\geqslant 25 \mathrm{mmHg}$ occurs in up to $70 \%$ of COPD patients and correlates with survival in this disease [4], there are currently no approved pharmacological treatment options for the pulmonary vascular disease. Only patients with suspected pulmonary arterial hypertension (PAH; group I of $\mathrm{PH}$ ) and concomitant mild COPD may be treated with specific vasoactive drugs [3]. Other forms of PH-COPD are treated with oxygen supplementation and anti-obstructive regimens according the Global Initiative for Chronic Obstructive Lung Disease guidelines, although patients with severe $\mathrm{PH}$ and moderate COPD (defined by $\mathrm{mPAP} \geqslant 35 \mathrm{mmHg}$ or $\mathrm{mPAP} \geqslant 25 \mathrm{mmHg}$ and cardiac index $<2.0 \mathrm{~L} \cdot \mathrm{min}^{-1} \cdot \mathrm{kg}^{-1}$ and forced expiratory volume in $1 \mathrm{~s}$ (FEV1) $<60 \%$ predicted) might benefit from vasodilatory treatment and thus should be considered in clinical trials of PH-COPD [5].

The lack of approved vasoactive therapy for PH-COPD is due to a controversy about the clinical efficacy of pulmonary vasoactive drugs and potential disturbance of ventilation-perfusion matching in the lung by vasodilatory agents, which may increase shunt flow through hypoventilated, hypoxic alveoli and thus increase hypoxaemia. Clinical trials with sildenafil showed conflicting results, possibly due to varying patient selection or COPD characterisation and different outcome parameters. While pulmonary vascular resistance (PVR) was decreased in a recent randomised controlled trial, functional parameters did not improve in most trials [6-8]. It has therefore been suggested that patients with circulatory but not ventilatory limitation during exercise might profit most from vasoactive treatment of $\mathrm{PH}$. However, large clinical trials are missing to answer the question of which patients profit most from therapy. Moreover, an approach addressing both vascular and alveolar remodelling as well as bronchial obstruction would be desirable. In this regard, it has been shown recently in animal models of smoke-induced emphysema that treatment with activators of the nitric oxide (NO)-cyclic guanosine monophosphate (cGMP) pathway can reduce $\mathrm{PH}$ and emphysema when applied in a preventive approach [9-11]. NO activates the soluble guanylate cyclase (sGC) to produce cGMP that activates cGMP-dependent protein kinases that can modulate apoptosis, proliferation, migration and extracellular matrix protein expression [9]. In these investigations tadalafil, an inhibitor of phosphodiesterase-5 (PDE5), which degrades cGMP, or riociguat or BAY 41-2272, stimulators of sGC, were applied concomitantly with smoke exposure of mice or guinea pigs, respectively. These compounds prevented the development of emphysema and $\mathrm{PH}[9,10]$. Riociguat also prevented inflammatory cell infiltration into the lung parenchyma, adhesion of neutrophils as well as peroxynitrite-induced apoptosis of alveolar and endothelial cells [9]. Moreover, the functionally essential sGC $\beta_{1}$-subunit was found to be downregulated in patients with COPD and in smoke-exposed mice [9]. These results suggest an important role of sGC signalling in the development of emphysema and $\mathrm{PH}$.

We thus investigated the treatment effect of sGC stimulation by riociguat in a therapeutic approach in smoke-exposed mice that develop emphysema and $\mathrm{PH}$, and retrospectively analysed the effect of riociguat treatment on $\mathrm{PH}$ in patients with severe $\mathrm{PH}$ and moderate COPD.

\section{Methods}

\section{Animals}

Adult male C57BL/6J mice, weight 20-22 g, age 3-4 months, were used for the experiments. For each group, 18 animals were randomly allocated to tobacco smoke exposure, room air exposure and treatment with riociguat or placebo (methylcellulose). All animals were analysed by haemodynamics, lung function testing and right heart morphometry. Additionally, the animals of each group were randomly assigned to be subjected to different analytical techniques: echocardiography was performed in $n=6$ animals per group, fluorescence molecular tomography-computer tomography (FMT-CT) in $n=5$ animals per group, histological analysis in $\mathrm{n}=10$ animals per group (for stereology $\mathrm{n}=5$ animals per group) and laser microdissection in $n=8$ animals per group. The $n$-numbers were predicted before the study by statistical analysis including dropout animals. The n-numbers of the results vary due to the dropout animals of the study resulting from technical reasons, e.g. complications during catheter placement in the animal. All animal experiments were approved by the governmental authorities (Regierungspräsidium Giessen).

\section{Tobacco smoke exposure and riociguat treatment in mice}

Mice were exposed to tobacco smoke and treated with riociguat as described previously [12]. Animals were sacrificed at 8 months (control and smoke exposed) or 11 months (curative approach). In the curative 
approach, animals were exposed to tobacco smoke for 8 months and tobacco smoke exposure was discontinued in the last 3 months of the treatment period. Riociguat was administered once per day over a period of 3 months via gavage, dissolved in $0.2 \mathrm{~mL} 2 \%$ methylcellulose at a dosage of 3 or $10 \mathrm{mg}^{-\mathrm{kg}^{-1}}$. In the placebo-treated groups $0.2 \mathrm{~mL} 2 \%$ methylcellulose was administered as placebo. Treatment was started after 8 months in control or smoke-exposed animals.

\section{Echocardiography and FMT-CT}

Echocardiography was performed 1 day prior to the final measurements under isoflurane inhalation anaesthesia as described previously [13]. FMT-CT used the matrix metalloproteinase (MMP) probe MMPSense 750 FAST (Perkin Elmer, Waltham, MA, USA) [14].

\section{In vivo measurements of lung function and haemodynamics}

Experiments were performed as described previously [12]. Lung function tests were performed using the flexiVent system (SCIREQ Scientific Respiratory Equipment, Montreal, QC, Canada). For haemodynamic measurements, a micro-tip catheter (SPR 671; Millar Instruments, Houston, TX, USA) was used. Afterwards the lungs and hearts were prepared for further analysis.

\section{Clinical analysis}

All investigations were performed according to the rules of Good Clinical Practice and approved by the University of Giessen Institutional Review Board (15/11 and 100/13). All patients gave their informed consent. Patients from the Giessen $\mathrm{PH}$ registry [15] treated with riociguat and diagnosed with severe $\mathrm{PH}-\mathrm{COPD}$ by right heart catheterisation maximally 6 months prior to riociguat start and subsequent right heart catheterisation maximally 7 months after start of riociguat were included in the retrospective analysis. PH-COPD was defined and treated according to the current recommendations of the Cologne Consensus Conference (moderate COPD defined as bronchial obstruction with a vital capacity/total lung capacity $<70 \%$ and $\mathrm{FEV} 1<60 \%$ predicted in combination with severe $\mathrm{PH}$ defined by $\mathrm{mPAP} \geqslant 35 \mathrm{mmHg}$ or $\mathrm{mPAP} \geqslant 25 \mathrm{mmHg}$ and cardiac index $\left.<2.0 \mathrm{~L} \cdot \mathrm{min}^{-1} \cdot \mathrm{kg}^{-1}\right)$. COPD was diagnosed by two respiratory medicine specialists evaluating positive smoking history and signs of emphysema, air trapping or chronic bronchitis on chest CT. FEV1 was $>30 \%$ predicted in all patients. All patients received riociguat based on current European or German guidelines [3,5].

\section{Statistics}

Statistical analyses were performed using Prism (GraphPad, La Jolla, CA, USA). All data are expressed as mean with standard error of the mean. Comparison between multiple groups was performed by ANOVA (one-way ANOVA, Dunett's multiple comparison test for comparison between control and treatment group, Tukey's post hoc test for comparison between different groups or Sidak's post hoc test for dependent variables). Comparison between oxygen saturation values and the interaction between riociguat treatment and exercise was performed by two-way ANOVA matching both factors with Tukey's post hoc test. The independent t-test was used for comparing equality of means between groups and the paired t-test for comparing the equality of means at different moments of time. p-values $<0.05$ were considered statistically significant. Normality and homoscedasticity were checked using residual diagnostics plots.

\section{Results}

Riociguat reverses $\mathrm{PH}$ in mice after 8 months of smoke exposure

Right ventricular systolic pressure (RVSP) was increased after 8 months of smoke exposure compared with controls (figure 1a: "Smoke exposure 8 months") and did not change upon subsequent exposure to room air for 3 months (figure 1a: "Smoke exposure 8 months +3 months placebo"). Riociguat at either concentration ( 3 or $10 \mathrm{mg} \cdot \mathrm{kg}^{-1} \cdot \mathrm{day}^{-1}$ ) decreased RVSP to the level of healthy lungs (figure 1a: "Smoke exposure 8 months +3 months 3 or $10 \mathrm{mg}$ riociguat"). Similarly, riociguat decreased systemic arterial pressure as well as left ventricular systolic pressure (supplementary figure E1a) in a dose-dependent manner and, at the high dose, even below levels of room air-exposed mice (figure 1b). As the mass of the left ventricle increased after smoke exposure plus room air exposure (supplementary figure E1b), right heart remodelling displayed as ratio of the weight of the right ventricle to the left ventricle plus septum was unchanged after smoke plus subsequent room air exposure (figure 1c), but right ventricular mass itself increased (supplementary figure E1c). Moreover, pulmonary vascular remodelling and mean vessel wall thickness were increased after 8 months of smoke exposure and 3 months of re-exposure to room air; both were reversed by treatment with the high dose of riociguat (figure 1d-f). Right ventricular internal diameter was increased after smoke exposure, indicating dilatation of the right ventricle due to right ventricular dysfunction, and decreased after riociguat treatment (figure 1g). Right heart function determined by tricuspid annular plane excursion (TAPSE) and the Tei index, as well as the cardiac index, was impaired after smoke plus room air exposure and could be restored by riociguat treatment (figure $1 \mathrm{~h}-\mathrm{j}$ ). 


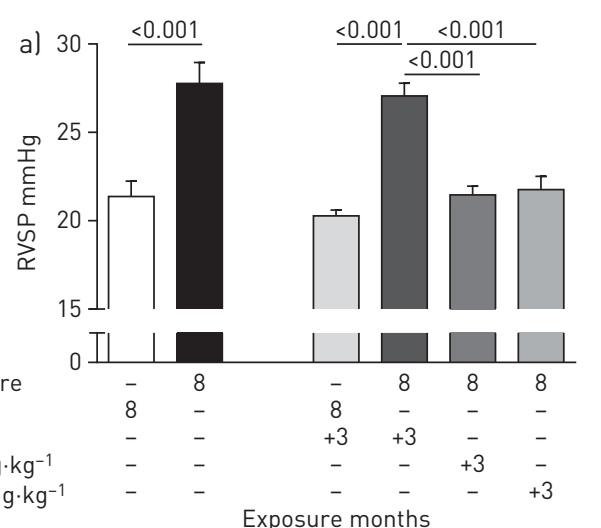

Exposure months

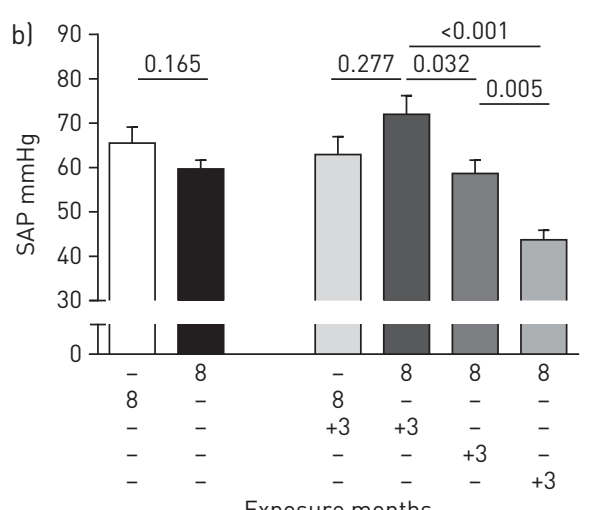

Exposure months

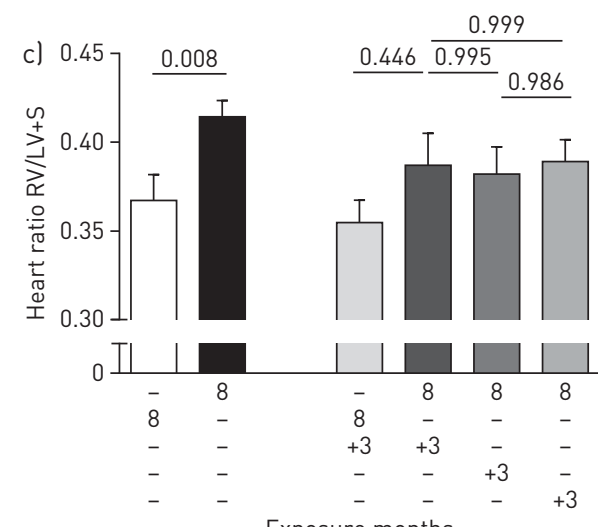

Exposure months
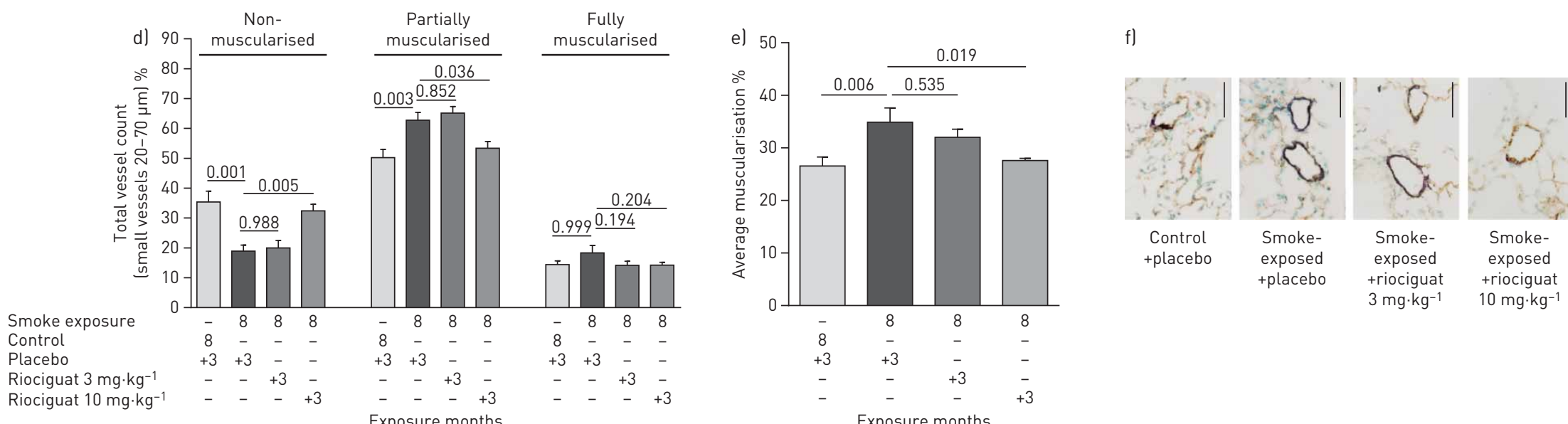

Riociguat $3 \mathrm{mg} \cdot \mathrm{kg}^{-1}$

Exposure months

Exposure months
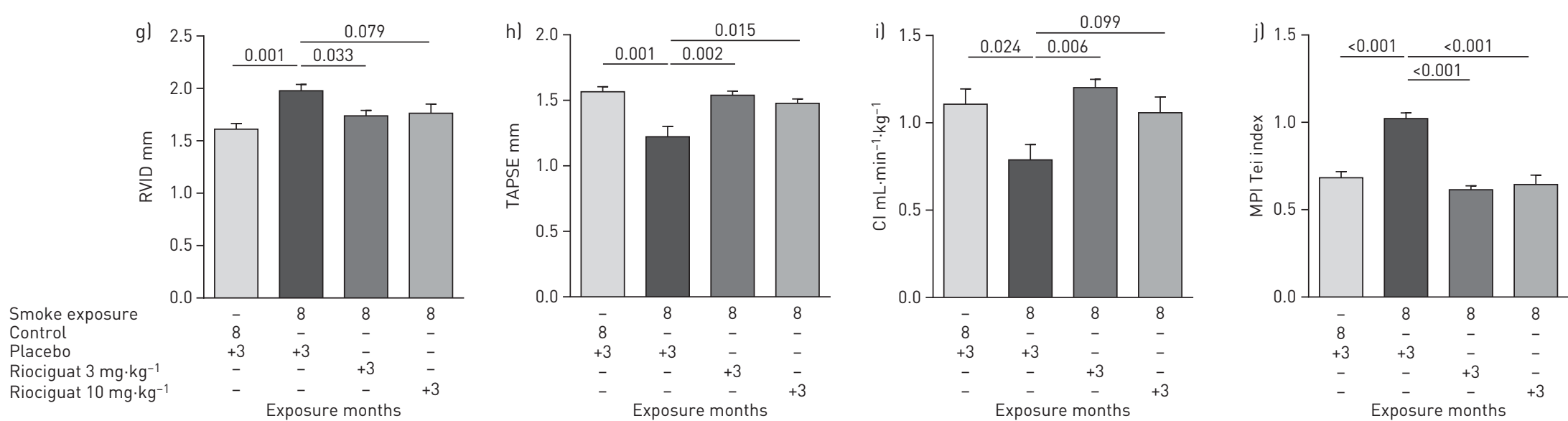
FIGURE 1 Reversal of cigarette smoke-induced pulmonary hypertension in mice treated with riociguat. RVSP: right ventricular systolic pressure; SAP: systemic arterial pressure; RV: right ventricle; LV+S: left ventricle plus septum; RVID: right ventricular inner diameter; TAPSE: tricuspid annular plane systolic excursion; $\mathrm{Cl}$ : cardiac index; MPI: myocardial performance index. a) RVSP ( $n=15-18$ per group), b) SAP ( $n=11-17$ per group) and c) right heart remodelling ( $n=14-18$ per group). Data are given for mice exposed for 8 months to cigarette smoke compared with the respective unexposed controls, as well as for mice exposed for 8 months to cigarette smoke and subsequently treated with either placebo or riociguat ( 3 or $10 \mathrm{mg} \cdot \mathrm{kg}^{-1}$ ) in room air conditions for 3 months (curative approach). $\mathrm{d}-\mathrm{f}$ ) Remodelling of the small vessels (20-70 $\mu$ m) expressed as d) percentage of total vessel count, given for non-, partially and fully muscularised vessels ( $\mathrm{n}=7-9$ per group), and e) average muscularisation of small vessels ( $n=7-9$ per group) in mice in the curative approach. $f$ ) Representative images of lung sections from mice in the curative approach, co-stained against $\alpha$-smooth muscle actin (purple) and von Willebrand factor (brown). Scale bar: $50 \mu m$. g-j) Echocardiography ( $n=4-6$ per group) showing g) RVID, h) TAPSE, i) $\mathrm{Cl}$ and j) MPI in mice in the curative approach. Data are presented as mean \pm SEM. $p$-values are indicated. The t-test was used for comparison between room air and cigarette smoke-exposed groups (a-c). One-way ANOVA (Dunett's multiple comparison post hoc test) was used for the groups in the curative approach.

\section{Riociguat attenuates pulmonary emphysema in mice after 8 months of smoke exposure}

Lung function measurement revealed an increase of the dynamic compliance after 8 months of smoke exposure (figure 2a: "Smoke exposure 8 months"), which was sustained even upon subsequent exposure to room air for 3 months (figure 2a: "Smoke exposure 8 months +3 months placebo"), but was not significantly affected by riociguat treatment (figure 2a: "Smoke exposure 8 months +3 months 3 or $10 \mathrm{mg}$ riociguat"). However, the pressure-volume loops indicate attenuation of emphysema development by riociguat treatment (figure $2 \mathrm{~b}$ ). Along these lines 1) air space and septal wall thickness were restored to values of room air-exposed mice by riociguat treatment (figure 2c-e), and 2) the number of alveoli that decreased after smoke exposure showed a tendency to increase after riociguat treatment $(\mathrm{p}=0.0537$ at $3 \mathrm{mg} \cdot \mathrm{kg}^{-1} \cdot \mathrm{day}^{-1}$ ) compared with placebo treatment (figure $2 \mathrm{f}$ and $\mathrm{g}$ ).

\section{Riociguat alters different signalling pathways in bronchi, pulmonary vessels and alveolar septa} Several intracellular signalling pathways were significantly altered in laser microdissected vessels and septa after treatment with riociguat compared with placebo-treated smoke-exposed mice (figure 3a). In particular, MMPs were highly downregulated in microdissected septa and vessels of riociguat-treated animals compared with placebo (figure $3 \mathrm{~b}-\mathrm{h}$ ). This regulation was confirmed in lung homogenate (supplementary figure E3a and $\mathrm{b}$ ). We thus next determined MMP activity in vivo by FMT-CT and found that cigarette smoke increased MMP activity which was attenuated in the riociguat-treated animals compared with placebo treatment (figure 3i). In line with this finding, upregulation of MMP activity was attenuated in lung homogenate and a human epithelial lung cell line (A549) in the presence of riociguat (supplementary figure E3c-e). Accordingly, reduction of proliferation of A549 cells, induced by cigarette smoke, could be rescued by riociguat as well as the cGMP analogue 8-bromo-cGMP, which directly activates protein kinase $\mathrm{G}(\mathrm{PKG})$ (supplementary figure E3f and $\mathrm{g}$ ).

As the inducible NO synthase (iNOS) plays an important role in the pathogenesis of smoke-induced emphysema, and iNOS inhibition was shown to reverse $\mathrm{PH}$ and emphysema in mice [12], we investigated the regulation of mRNA expression of iNOS. As expected, iNOS was strongly upregulated by smoking; riociguat was found to significantly downregulate iNOS mRNA in lung homogenate of mice exposed to smoke and subsequently room air (figure 4a). In line with this finding, nitrotyrosine was increased after smoke exposure and returned to previous levels by riociguat treatment (figure $4 \mathrm{~b}$ and $\mathrm{c}$ ).

\section{Treatment with riociguat in PH patients with COPD improves pulmonary haemodynamics}

Retrospective analysis of seven PH-COPD patients showed a significant decrease of PVR after treatment with riociguat for 4-7 months, while blood gases and lung function parameters did not change significantly (table 1). Oxygen saturation dropped after exercise compared with before exercise. However, no significant difference was found in the magnitude of the drop when comparing values prior to with those during riociguat treatment. Nevertheless, there was a tendency for lower oxygen saturation levels after exercise during riociguat treatment compared with before riociguat treatment (supplementary figure E4). Of note, pulmonary arterial wedge pressure (PAWP) did not increase during riociguat treatment. One patient stopped riociguat treatment after 3 months. MMP9 levels were determined in six of the patients, which decreased significantly during riociguat treatment (figure 5). Noninvasive long-term follow-up of these patients showed a decrease of the airway resistance without change in oxygenation. Similar results were obtained in three only noninvasively followed patients (supplementary table E1).

\section{Discussion}

Our study investigated for the first time the therapeutic potential of riociguat in PH-COPD in a translational approach. We showed that riociguat can reverse $\mathrm{PH}$ and partially reverse established emphysema in a mouse model of long-term tobacco smoke exposure. In agreement with these findings, riociguat altered intracellular signalling pathways mainly related to inflammatory and proliferative 


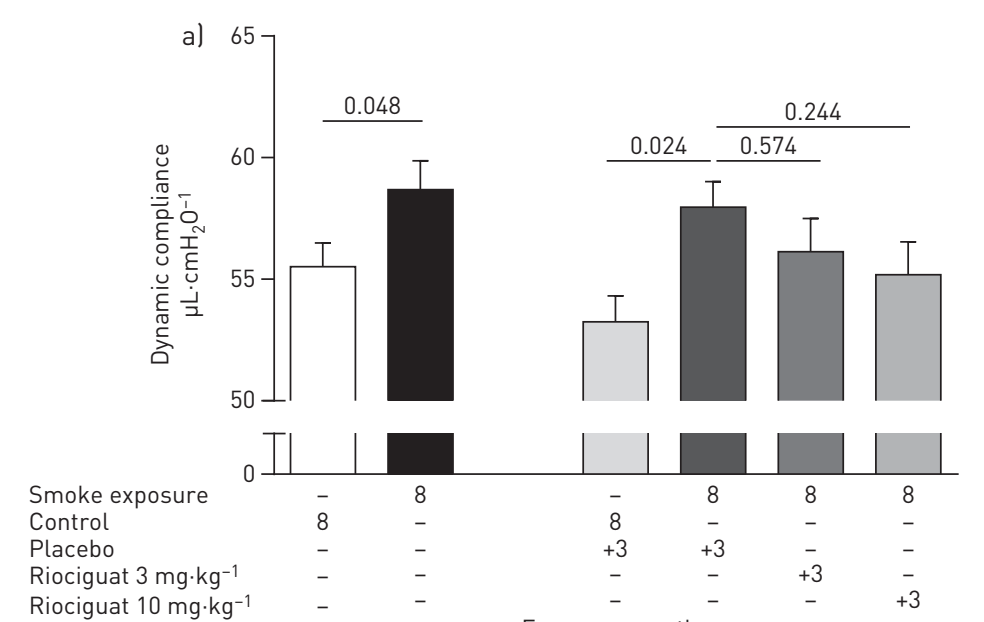

Riociguat $10 \mathrm{mg} \cdot \mathrm{kg}^{-1}$

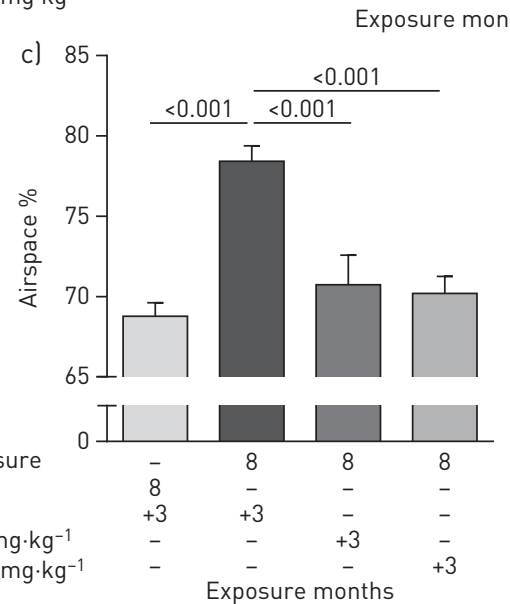

Control

Riociguat $3 \mathrm{mg} \cdot \mathrm{kg}^{-1}$

Exposure months

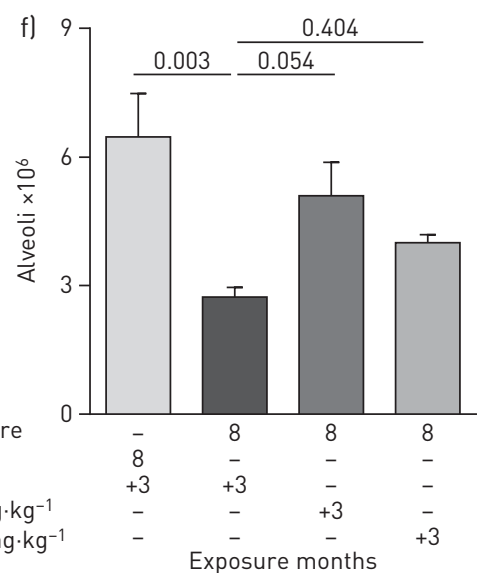

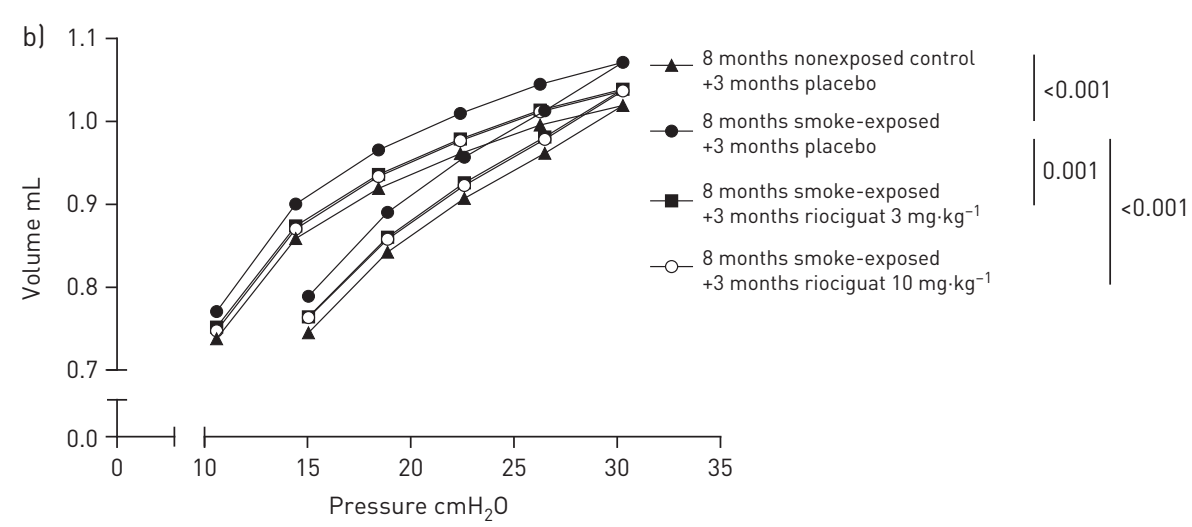
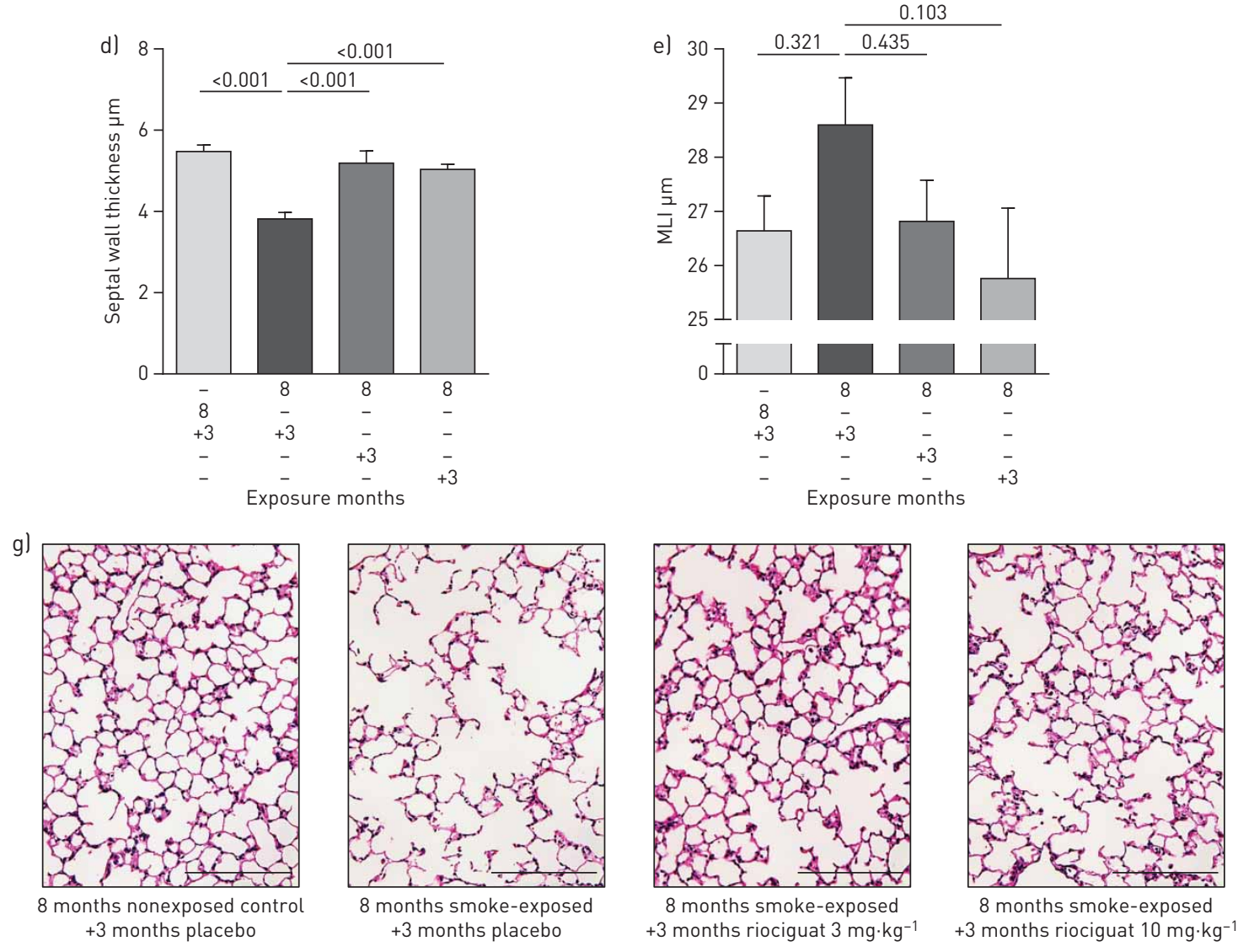
FIGURE 2 Partial reversal of cigarette smoke-induced pulmonary emphysema in mice treated with riociguat. MLI: mean linear intercept. a) Lung function determined as dynamic compliance ( $n=14-18$ per group) in mice exposed for 8 months to cigarette smoke compared with the respective unexposed controls, as well as in mice exposed for 8 months to cigarette smoke and subsequently treated with either placebo or riociguat $(3$ or $10 \mathrm{mg} \cdot \mathrm{kg}^{-1}$ ) in room air conditions for 3 months (curative approach). b) Respiratory pressure-volume loops ( $\mathrm{n}=11-15$ per group) in mice in the curative approach. $c-$ e) Alveolar morphometry ( $n=8-10)$ showing c) percentage airspace, d) septal wall thickness and e) MLI in mice in the curative approach. f) Number of alveoli determined by stereology ( $n=5$ per group). g) Representative images (haematoxylin and eosin staining) of lungs from mice in the curative approach. Scale bar: $200 \mu \mathrm{m}$. Data are presented as mean \pm SEM. p-values are indicated. The t-test was used for comparison between room air and cigarette smoke-exposed groups (a). One-way ANOVA (Dunett's multiple comparison post hoc test) was used for the groups in the curative approach.

signalling. Moreover, our data show that riociguat treatment improved $\mathrm{PH}$ in patients with COPD and $\mathrm{PH}$ without worsening of oxygenation.

In line with our findings, previous studies showed that enhancement of the NO-cGMP signalling pathway by preventive treatment with sGC stimulators or inhibitors of PDE5, which degrades cGMP, can inhibit development of $\mathrm{PH}$ and emphysema in animal models of smoke-induced emphysema [9-11]. Going beyond these studies, we now show for the first time in a curative approach that the sGC stimulator riociguat could also attenuate $\mathrm{PH}$ and emphysema when treatment was started after full establishment of the disease. In this model neither PH nor emphysema is spontaneously reversible after cessation of smoke exposure, as shown previously [12] and confirmed again in our current study by showing that RVSP and dynamic compliance were increased to a similar degree in mice exposed for 8 months to smoke or in mice exposed for 8 months to smoke and subsequently for 3 months to room air. We show that the alterations in RVSP and right heart function, as well as pulmonary vascular remodelling in the high-dose treatment group, could be reversed by riociguat treatment. Of note, systemic arterial pressure also decreased by treatment with riociguat. With regard to emphysema, we showed persistent alterations of the dynamic compliance and lung structure 3 months after re-exposure to room air under placebo treatment. Treatment with riociguat reversed alterations in air space and septal wall thickness, and tended to restore the number of alveoli.

In a preventive approach, it was previously shown that sGC stimulators and PDE5 inhibition reduced cigarette smoke-induced pulmonary inflammation $[9,10]$. Moreover, sGC stimulators and cGMP analogues inhibited the induction of apoptosis of alveolar and endothelial cells by peroxynitrite, which plays an important role in the development of emphysema [10]. Such mechanisms may also contribute to lung regeneration by enhancing the lung maintenance programme [12]. In line with this suggestion, we now show in the curative approach that riociguat affected various signalling pathways related to inflammatory responses and lung development such as fibroblast growth factor-10 [16] and MMPs [17]. In particular, loss of MMP12 can protect mice from smoke-induced lung disease [17]. In human COPD, but also PH, MMP9 was found to be upregulated [18, 19]. Riociguat may attenuate smoke-induced $\mathrm{PH}$ and emphysema by decreasing MMPs, as we found decreased MMP9 and MMP12 mRNA levels, as well as attenuation of a smoke-induced increase of MMP activity in vivo (detected by FMT-CT) after riociguat treatment in mice. Of course, one has to be generally cautious when transferring findings from mice to humans. However, the fact that circulating MMP9 levels were decreased in PH patients with COPD after 4-7 months treatment with riociguat is very much in line with the findings in mice. This is, to the best of our knowledge, the first report that provides strong evidence that riociguat decreases the expression and activity of MMPs in the context of emphysema, while previous reports show both an upregulation and a downregulation of MMPs by activation of NO-cGMP signalling depending on the investigated cell type and pathological condition [20]. Increased cGMP can downregulate MMP9 expression by PKG-dependent signalling and reduce the stability of MMP9 mRNA [20]. In support of this suggestion, we show here that a direct stimulator of $\mathrm{PKG}$, i.e. 8-bromo-cGMP, restored the smoke-driven downregulation of proliferation in a human lung epithelial cell line.

Most interestingly, riociguat treatment also downregulated iNOS expression and nitrotyrosine levels in the mouse model. This can be caused by the anti-inflammatory effects of riociguat [21] and explain the regenerative effect, as iNOS inhibition has recently been shown to regenerate the lung after smoke-induced $\mathrm{PH}$ and emphysema [12]. In this regard, it is well known that excessive NO production by iNOS has deleterious effects on cellular survival, while low amounts of NO production by exogenous NOS, which activates cGMP-dependent mechanisms, promotes cell survival [22]. The effect of riociguat on iNOS expression observed here has, to the best of our knowledge, not been described for lung regeneration before.

Despite the promising results of sGC stimulators and PDE5 inhibitors in animal models of $\mathrm{PH}$ and emphysema, the treatment effect in human COPD remains unclear. Treatment of PH-COPD is limited by the fact that pulmonary vasodilation may affect ventilation-perfusion matching and thus arterial 


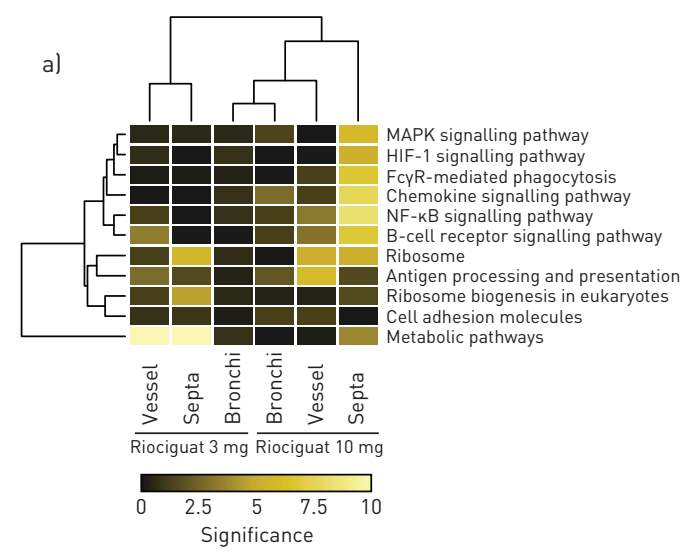

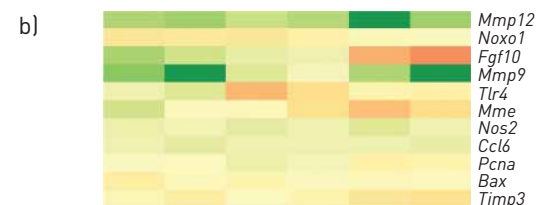

Riociguat $3 \mathrm{mg} 10 \mathrm{mg} 3 \mathrm{mg} 10 \mathrm{mg} 3 \mathrm{mg} 10 \mathrm{mg}$

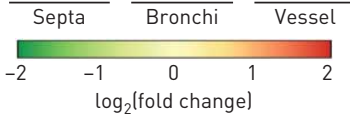

i)
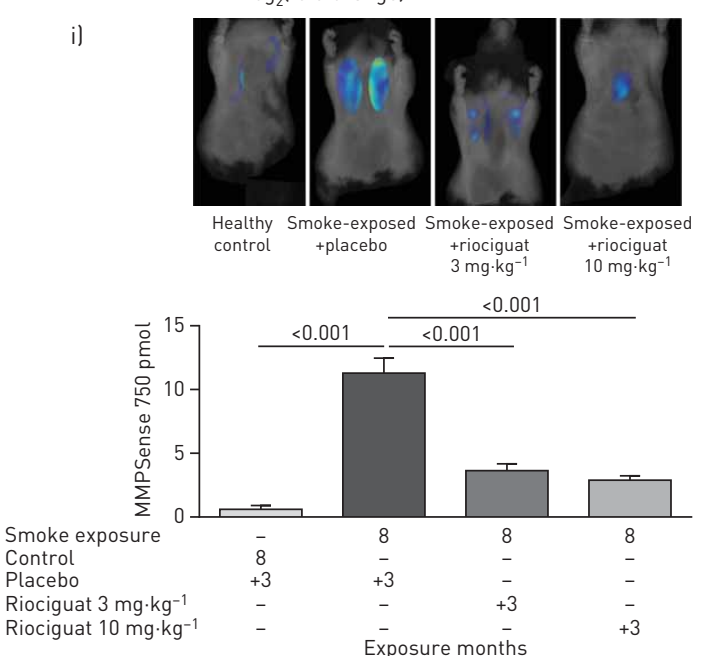

c)
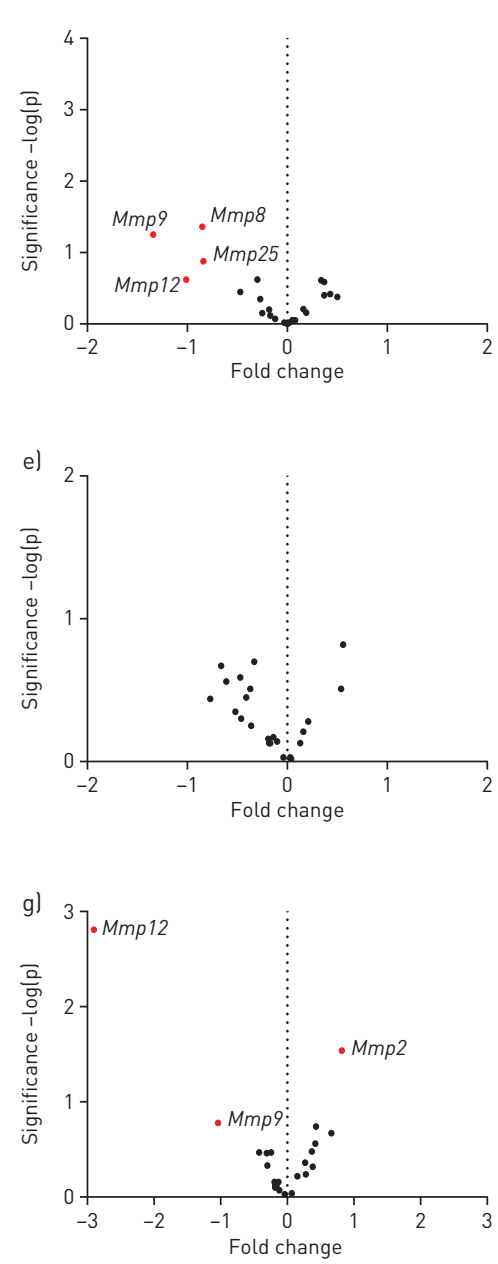

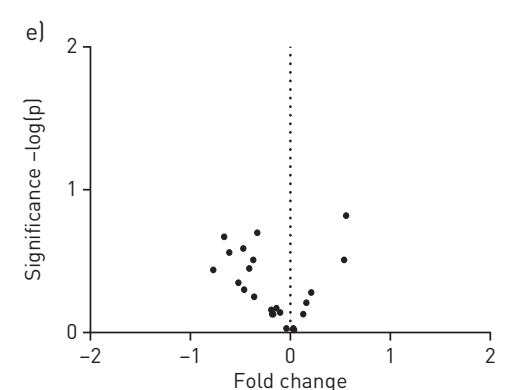

d)
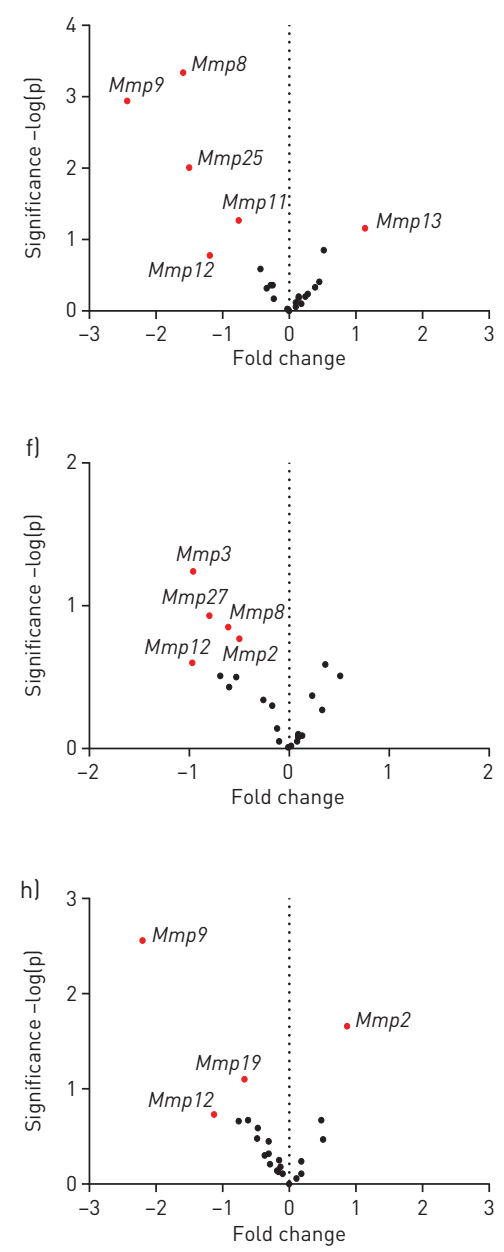

FIGURE 3 Microarray gene expression analysis of laser microdissected vessels, bronchi and septa, and in vivo matrix metalloproteinase (MMP) activity. MAPK: mitogen-activated protein kinase; HIF-1: hypoxia inducible factor-1; Mmp12: MMP12; Noxo1: nicotinamide adenine dinucleotide phosphate oxidase organiser-1; Fgf10: fibroblast growth factor-10; Mmp9: MMP9; Tlr4: Toll-like receptor-4; Mme: membrane metallopeptidase; Nos2: nitric oxide synthase-2; Ccl6: chemokine (C-C motif) ligand-6; Pcna: proliferating cell nuclear antigen; Bax: B-cell lymphoma-2 associated X protein; Timp3: MMP inhibitor-3. a) Heat map showing significant alterations of different signalling pathways or b) expression changes (log 2 (fold change)) of different genes in vessels, septa and bronchi of mice exposed to cigarette smoke for 8 months and subsequently treated with 3 or $10 \mathrm{mg} \cdot \mathrm{kg}^{-1} \cdot \mathrm{day}^{-1}$ riociguat for 3 months in room air conditions compared with mice exposed to cigarette smoke for 8 months and subsequently treated with placebo for 3 months in room air conditions. The values given display the difference of verum (riociguat treated) versus control (placebo treated). c-h) Volcano plots showing differences in expression levels of MMPs between c, e, g) riociguat $3 \mathrm{mg}^{\circ} \mathrm{kg}^{-1} \cdot \mathrm{day}^{-1}$ and d, $\mathrm{f}, \mathrm{h}$ ) riociguat $10 \mathrm{mg} \cdot \mathrm{kg}^{-1}$. day ${ }^{-1}$ versus placebo treatment animals in c, d) alveolar septa, e, f) bronchi and $\mathrm{g}$, h) vessels (red symbols show MMPs with the most significant changes). i) In vivo assessment of MMP activity by the fluorescence molecular tomography imaging system, detected with the MMPSense probe, representing activity of MMP2, 3, 7, 9, 12 and $13(n=4-5)$. Data are presented as mean \pm SEM. p-values are indicated. One-way ANOVA (Dunett's multiple comparison test) was used for groups in the curative approach; comparison was done between the cigarette smoke-exposed group treated with placebo and each other group.

oxygenation. Moreover, the ventilatory limitation due to bronchial obstruction and hyperinflation, as well as decreased oxygen exchange capacity due to emphysema, may compromise the exercise capacity of patients to a larger extent than the circulatory limitation caused by $\mathrm{PH}$. Thus, current European guidelines only recommend treatment in patients with severe $\mathrm{PH}$ and mild COPD [3]. However, the treatment effect in patients with severe $\mathrm{PH}$ and moderate COPD defined according to the national recommendations of the Cologne Consensus Conference remains unclear [5]. 


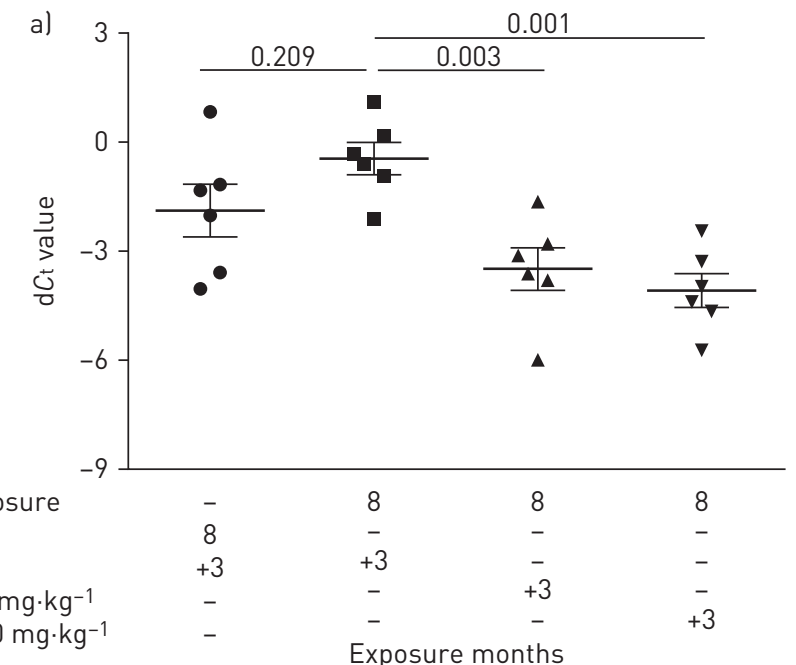

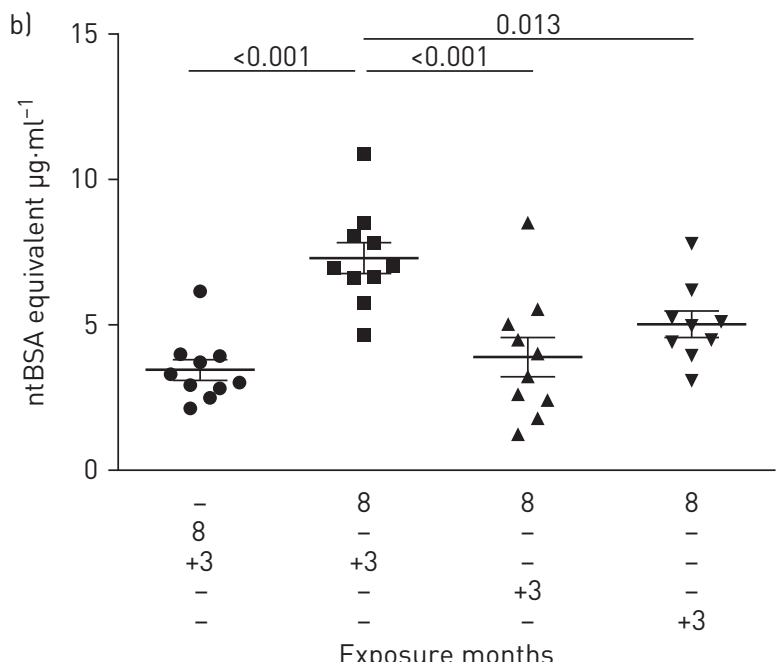

Exposure months c)
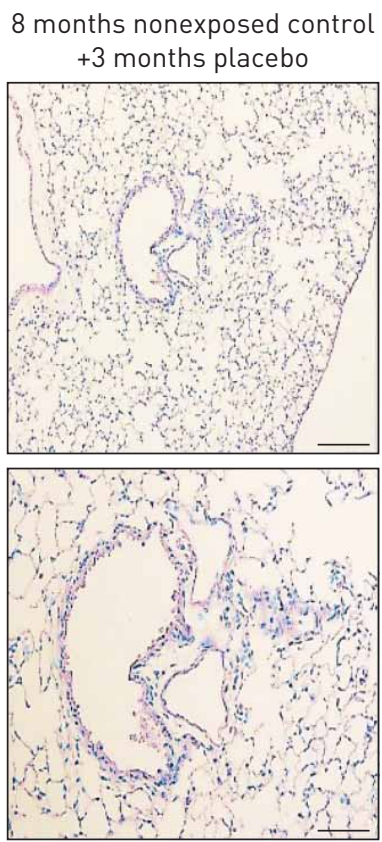

8 months smoke-exposed +3 months placebo
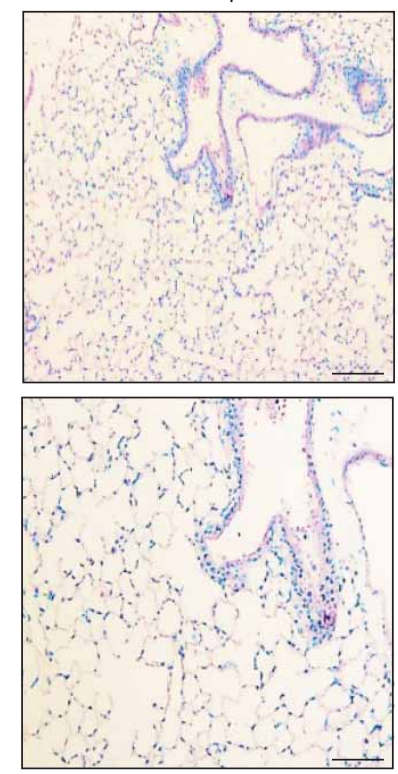

8 months smoke-exposed +3 months riociguat $3 \mathrm{mg} \cdot \mathrm{kg}^{-1}$
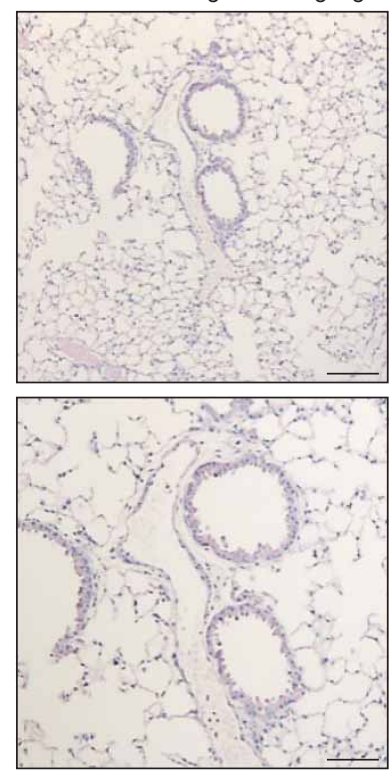

8 months smoke-exposed +3 months riociguat $10 \mathrm{mg} \cdot \mathrm{kg}^{-1}$
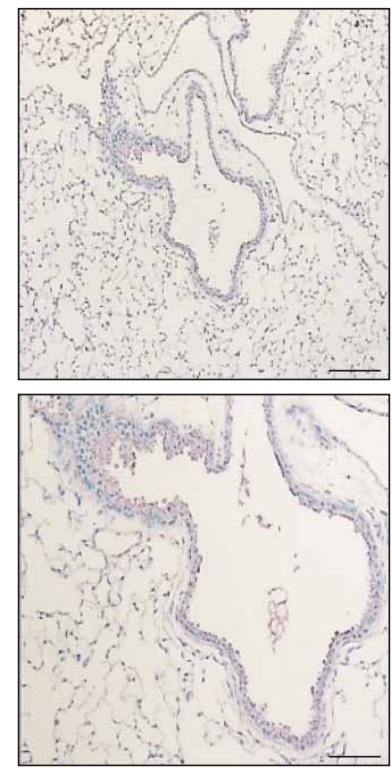

FIGURE 4 Effect of riociguat on inducible nitric oxide synthase (iNOS) expression and nitrotyrosine formation in mouse lungs. Ct: cycle threshold; ntBSA: nitrated bovine serum albumin. a) Expression of iNOS in lung homogenate of animals in the curative approach ( $n=6$ ), assessed using quantitative PCR. b) Nitrotyrosine formation, quantified in lung homogenate ( $n=9-10)$ by ELISA, expressed as equivalent of ntBSA standard. Data are presented as mean士SEM. p-values are indicated. One-way ANOVA (Dunett's multiple comparison test) was used for groups in the curative approach; comparison was done between the cigarette smoke-exposed group treated with placebo and each other group. c) Representative images of immunohistochemistry staining against nitrotyrosine residues (blue). Scale bar: $50 \mu \mathrm{m}$.

As peripheral airway obstruction is common in idiopathic $\mathrm{PAH}[23,24]$ and has also been observed in chronic thromboembolic PH (CTEPH) [25], bronchial obstruction in our patients may occur independently from COPD. COPD was thus diagnosed according to smoking history and radiology findings. The analysis of the haemodynamic data showed that after treatment with riociguat (up to 7 months) PVR decreased significantly in PH patients with COPD. The decrease was in the range of previously reported changes in PVR during riociguat treatment in PAH and CTEPH [26-28]. These findings are also in line with a recent randomised controlled trial with sildenafil in PH-COPD that showed an improvement in haemodynamics but not functional capacity after treatment [6]. Of note, we could not find any deterioration of oxygenation in COPD patients. These findings are consistent with a previous study with a single application of riociguat, which showed improvement of haemodynamics and stable oxygenation [29]. These observations can be explained by the fact that shunt flow is increased in chronic bronchitis and thus may not be enhanced by vasodilatory treatment [30], so that patients with a COPD 
TABLE 1 Haemodynamics and lung function in pulmonary hypertension-associated chronic obstructive pulmonary disease in the seven patients treated with riociguat

\section{Pre-treatment}

\section{Post-treatment \\ 14-7 months after start of riociguat)}

\section{p-value \\ p-value}

$46 \pm 4$
$11 \pm 2$
$2.25 \pm 0.16$
$681 \pm 143$
$1511 \pm 186$
$65 \pm 4$
$36 \pm 4$
$2.14 \pm 1.16$
$209 \pm 53$
$46 \pm 3$
$53 \pm 4$
$55 \pm 3$
$44 \pm 5$
$269 \pm 28$
$94 \pm 1$
$91 \pm 2$
$250 \pm 91$
$79 \pm 3$
$20 \pm 2$

$\begin{array}{cc}38 \pm 3 & 1.000 \\ 11 \pm 1 & 1.000 \\ 3.02 \pm 0.20 & 1.000 \\ 389 \pm 68 & <0.001^{*} \\ 931 \pm 116 & <0.001^{*}\end{array}$

$\begin{array}{cc}75 \pm 2 & 0.901 \\ 37 \pm 3 & 0.998 \\ 2.00 \pm 0.79 & 1.000 \\ 198 \pm 51 & 0.886 \\ 48 \pm 5 & 0.998 \\ 57 \pm 6 & 0.999 \\ 57 \pm 3 & 0.998 \\ 43 \pm 5 & 0.999 \\ & \\ 269 \pm 56 & 1.000 \\ 95 \pm 1 & 1.000 \\ 84 \pm 2 & 1.000 \\ 118 \pm 61 & <0.001^{*}\end{array}$

$75 \pm 9$

$22 \pm 2$
0.989

0.997
Post-treatment

19-24 months after start of riociguat)

Data are presented as mean \pm SEM, unless otherwise stated. mPAP: mean pulmonary arterial pressure; PAWP: pulmonary arterial wedge

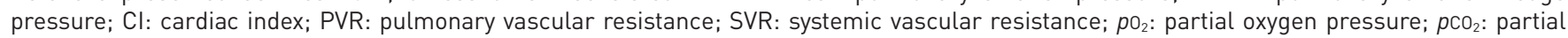
carbon dioxide pressure; Rtot: total airway resistance; FEV1: forced expiratory volume in 1 s; VC: vital capacity; RV: residual volume; TLC: total lung capacity; DLco: diffusing capacity of the lung for carbon monoxide; 6MWD: 6-min walk distance; $\mathrm{SO}_{2}$ pre/post: oxygen saturation before/ after 6-min walk test; BNP: brain natriuretic peptide; sPAP: systolic pulmonary arterial pressure; TAPSE: tricuspid annular plane systolic excursion. ${ }^{\#}: \mathrm{n}=4 ;{ }^{\natural}: \mathrm{n}=5$ (time-points of BNP vary up to 3 months from the time-points of the other parameters). All $p$-values are provided for comparison to pre-treatment groups unless otherwise indicated. *: significant difference compared with pre-treatment; **: significant difference compared with first time-point post-treatment.

phenotype of chronic bronchitis may suffer less from deoxygenation during vasodilatory treatment than patients with pulmonary emphysema and low shunt flow. Although emphysema-induced vessel loss has been described as a pathomechanism in COPD-PH [31], $\mathrm{PH}$ has been shown to correlate with small airway remodelling and thus potentially the extent of chronic bronchitis [32]. However, the situation may be different under exercise, as oxygen saturation tended to decrease more after the 6-min walk test under riociguat treatment than before riociguat treatment, reaching levels in some patients that require adaptation of the oxygen supply. This aspect should be taken into account in further randomised trials when investigating the effect of vasodilator treatment in emphysema.

We could not find a difference in increase of PAWP that could have been expected, as cardiac dysfunction due to hyperinflation has been recently described in COPD patients [33] and pre-capillary vasodilation may lead to pulmonary venous congestion in left heart dysfunction. However, left heart function may have also been promoted by a decrease in systemic pressure, as systemic vascular resistance was also decreased in our patients, similar to the animal model in which riociguat decreased the systemic vascular pressure. In line with our findings, systemic arterial pressure was significantly decreased in two large clinical trials with riociguat in PAH or systolic left ventricular dysfunction [28, 34], but PAWP was unchanged [34].

We observed a decrease in airway resistance after treatment with riociguat and a tendency for improved $\mathrm{FEV} 1 /$ vital capacity ratios. Decreased bronchial obstruction may be related to improved pulmonary haemodynamics as airway obstruction was described in idiopathic PAH patients and may be caused by a lack of vasodilators that also cause bronchodilation, e.g. NO [23]. Moreover, riociguat may affect bronchial hyperresponsiveness, as it was recently shown that PDE5 inhibition can decrease methacholine-induced bronchial obstruction [35]. 


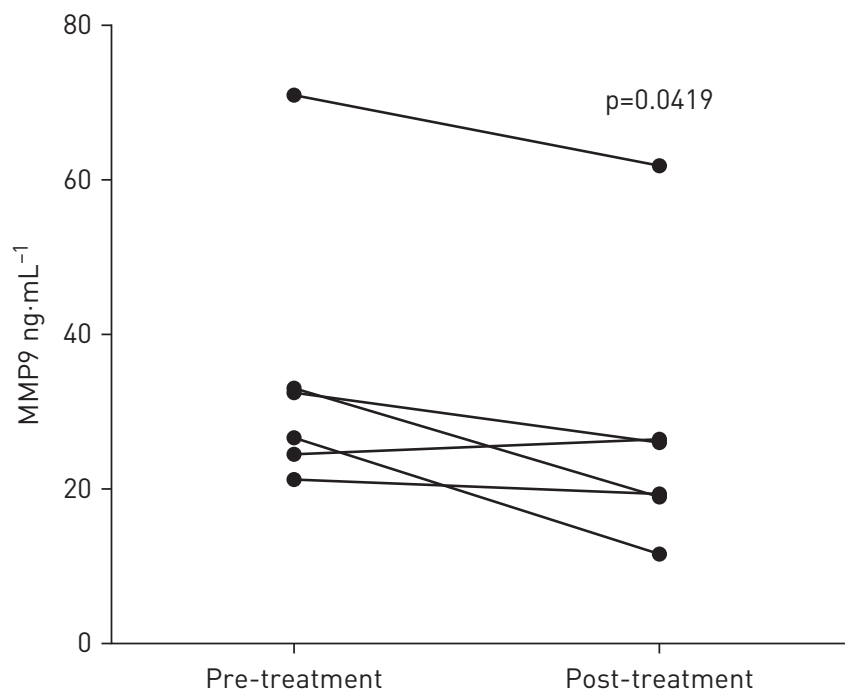

FIGURE 5 Effect of riociguat on matrix metalloproteinase-9 (MMP9) in patients. Blood serum concentration of MMP9 in patients with chronic obstructive pulmonary disease before and 3-7 months after start of riociguat treatment $(n=6)$.

Our study is limited by the low number of patients included. This is due to the fact that in the past only few patients with $\mathrm{PH}-\mathrm{COPD}$ were treated with riociguat as the adverse effects were unclear. However, the effects of riociguat in COPD patients reported here were quite consistent, even in the low numbers. Thus, this study allows at least preliminary insight into the effects of riociguat treatment in $\mathrm{PH}$ patients with COPD. A further limitation of our study is the fact that changes in concomitant therapy of COPD were not taken into account. However, COPD was treated according to current guidelines and therefore optimal bronchodilatory therapy was given at any time in the retrospective analysis.

In conclusion, riociguat may be beneficial for treatment of $\mathrm{PH}$ in COPD patients. However, it remains to be clarified which patients profit functionally from vasodilatory therapy in general and if additional effects on progression of COPD can be observed.

Acknowledgements: The authors thank M. Wessendorf, K. Homberger, I. Breitenborn-Mueller, C. Vroom and K. Quanz (Justus Liebig University, Giessen, Germany) for technical assistance.

Conflict of interest: A. Pichl has nothing to disclose. N. Sommer has nothing to disclose. M. Bednorz has nothing to disclose. M. Seimetz has nothing to disclose. S. Hadzic has nothing to disclose. S. Kuhnert reports nonfinancial (travel) support from Teva, Bayer and Astellas, personal fees for advisory board work from Chiesi, personal fees for advisory board work and nonfinancial (travel) support from GSK, personal fees for advisory board work, honoraria and nonfinancial (travel) support from Novartis, honoraria from Berlin-Chemie Menarini and AstraZeneca, outside the submitted work. S. Kraut has nothing to disclose. E.T. Roxlau has nothing to disclose. B. Kojonazarov has nothing to disclose. J. Wilhelm has nothing to disclose. M. Gredic has nothing to disclose. H. Gall reports grants and personal fees from Actelion, AstraZeneca, Bayer, BMS, GSK, Janssen Cilag, Lilly, MSD, Novartis, Pfizer, and United Therapeutics/ OMT, outside the submitted work. K. Tello has nothing to disclose. M.J. Richter reports grants from United Therapeutics, grants and personal fees for consultancy and lecturing from Bayer, personal fees for lecturing from Actelion, Mundipharma, Roche and OMT, outside the submitted work. O. Pak has nothing to disclose. A. Petrovic has nothing to disclose. M. Hecker has nothing to disclose. R.T. Schermuly has nothing to disclose. F. Grimminger has nothing to disclose. W. Seeger has received honoraria for consultant activities from Bayer, United Therapeutics and Liquidia, outside the submitted work. H.A. Ghofrani reports personal fees for advisory board work from Actelion, Bayer, GSK, Novartis and Pfizer, personal fees for consultancy from Actelion, Bayer, GSK, MSD, Novartis and Pfizer, personal fees for lecturing from Actelion, Bayer, GSK, Novartis and Pfizer, personal fees for meeting attendance from Actelion, Bayer, GSK, MSD, Novartis and Pfizer, and grants from Deutsche Forschungsgemeinschaft (DFG), outside the submitted work. N. Weissmann has nothing to disclose.

Support statement: Funded by the Deutsche Forschungsgemeinschaft (German Research Foundation): 268555672SFB1213, A07, CP02. Funding information for this article has been deposited with the Crossref Funder Registry.

\section{References}

1 Vestbo J, Hurd SS, Agusti AG, et al. Global strategy for the diagnosis, management, and prevention of chronic obstructive pulmonary disease: GOLD executive summary. Am J Respir Crit Care Med 2013; 187: 347-365.

2 Peinado VI, Pizarro S, Barbera JA. Pulmonary vascular involvement in COPD. Chest 2008; 134: 808-814.

3 Galiè N, Humbert M, Vachiery JL, et al. 2015 ESC/ERS Guidelines for the diagnosis and treatment of pulmonary hypertension: The Joint Task Force for the Diagnosis and Treatment of Pulmonary Hypertension of the European 
Society of Cardiology (ESC) and the European Respiratory Society (ERS): Endorsed by: Association for European Paediatric and Congenital Cardiology (AEPC), International Society for Heart and Lung Transplantation (ISHLT). Eur Respir J 2015; 46: 903-975.

4 Minai OA, Chaouat A, Adnot S. Pulmonary hypertension in COPD: epidemiology, significance, and management: pulmonary vascular disease: the global perspective. Chest 2010; 137: 39s-51s.

5 Olschewski H, Behr J, Bremer H, et al. Pulmonale Hypertonie bei Lungenkrankheiten: Empfehlungen der Kolner Konsensus-Konferenz 2016. [Pulmonary hypertension due to chronic lung disease: recommendations of the Cologne Consensus Conference 2016.] Dtsch Med Wochenschr 2016; 141: S57-S61.

6 Vitulo P, Stanziola A, Confalonieri M, et al. Sildenafil in severe pulmonary hypertension associated with chronic obstructive pulmonary disease: a randomized controlled multicenter clinical trial. J Heart Lung Transplant 2017; 36: $166-174$.

7 Rao RS, Singh S, Sharma BB, et al. Sildenafil improves six-minute walk distance in chronic obstructive pulmonary disease: a randomised, double-blind, placebo-controlled trial. Indian I Chest Dis Allied Sci 2011; 53: 81-85.

8 Blanco I, Santos S, Gea J, et al. Sildenafil to improve respiratory rehabilitation outcomes in COPD: a controlled trial. Eur Respir J 2013; 42: 982-992.

9 Weissmann N, Lobo B, Pichl A, et al. Stimulation of soluble guanylate cyclase prevents cigarette smoke-induced pulmonary hypertension and emphysema. Am J Respir Crit Care Med 2014; 189: 1359-1373.

10 Seimetz M, Parajuli N, Pichl A, et al. Cigarette smoke-induced emphysema and pulmonary hypertension can be prevented by phosphodiesterase 4 and 5 inhibition in mice. PLoS One 2015; 10: e0129327.

11 Dominguez-Fandos D, Valdes C, Ferrer E, et al. Sildenafil in a cigarette smoke-induced model of COPD in the guinea-pig. Eur Respir J 2015; 46: 346-354.

12 Seimetz M, Parajuli N, Pichl A, et al. Inducible NOS inhibition reverses tobacco-smoke-induced emphysema and pulmonary hypertension in mice. Cell 2011; 147: 293-305.

13 Pullamsetti SS, Kojonazarov B, Storn S, et al. Lung cancer-associated pulmonary hypertension: role of microenvironmental inflammation based on tumor cell-immune cell cross-talk. Sci Transl Med 2017; 9: eaai9048.

14 Sibinska Z, Tian X, Korfei M, et al. Amplified canonical transforming growth factor-beta signalling via heat shock protein 90 in pulmonary fibrosis. Eur Respir J 2017; 49: 1501941.

15 Gall H, Felix JF, Schneck FK, et al. The Giessen Pulmonary Hypertension Registry: survival in pulmonary hypertension subgroups. J Heart Lung Transplant 2017; 36: 957-967.

16 Sekine K, Ohuchi H, Fujiwara M, et al. Fgf10 is essential for limb and lung formation. Nat Genet 1999; 21: 138-141.

17 Hendrix AY, Kheradmand F. The role of matrix metalloproteinases in development, repair, and destruction of the lungs. Prog Mol Biol Transl Sci 2017; 148: 1-29.

18 Navratilova Z, Kolek V, Petrek M. Matrix metalloproteinases and their inhibitors in chronic obstructive pulmonary disease. Arch Immunol Ther Exp 2016; 64: 177-193.

19 Chelladurai P, Seeger W, Pullamsetti SS. Matrix metalloproteinases and their inhibitors in pulmonary hypertension. Eur Respir J 2012; 40: 766-782.

20 O'Sullivan S, Medina C, Ledwidge M, et al. Nitric oxide-matrix metaloproteinase-9 interactions: biological and pharmacological significance: NO and MMP-9 interactions. Biochim Biophys Acta 2014; 1843: 603-617.

21 Ahluwalia A, Foster P, Scotland RS, et al. Antiinflammatory activity of soluble guanylate cyclase: cGMP-dependent down-regulation of P-selectin expression and leukocyte recruitment. Proc Natl Acad Sci USA 2004; 101: $1386-1391$

22 Forstermann U, Sessa WC. Nitric oxide synthases: regulation and function. Eur Heart J 2012; 33: 829-837.

23 Low AT, Medford AR, Millar AB, et al. Lung function in pulmonary hypertension. Respir Med 2015; 109: 1244-1249.

24 Meyer FJ, Ewert R, Hoeper MM, et al. Peripheral airway obstruction in primary pulmonary hypertension. Thorax 2002; 57: 473-476.

25 Fukushi K, Kataoka M, Shimura N, et al. Impaired respiratory function in chronic thromboembolic pulmonary hypertension: a comparative study with healthy control subjects. Ann Am Thorac Soc 2016; 13: 1183-1184.

26 Ghofrani HA, D'Armini AM, Grimminger F, et al. Riociguat for the treatment of chronic thromboembolic pulmonary hypertension. N Engl J Med 2013; 369: 319-329.

27 Ghofrani HA, Galie N, Grimminger F, et al. Riociguat for the treatment of pulmonary arterial hypertension. N Engl J Med 2013; 369: 330-340.

28 Galie N, Grimminger F, Grunig E, et al. Comparison of hemodynamic parameters in treatment-naive and pre-treated patients with pulmonary arterial hypertension in the randomized phase III PATENT-1 study. $J$ Heart Lung Transplant 2017; 36: 509-519.

29 Ghofrani HA, Staehler G, Grunig E, et al. Acute effects of riociguat in borderline or manifest pulmonary hypertension associated with chronic obstructive pulmonary disease. Pulm Circ 2015; 5: 296-304.

30 Kent BD, Mitchell PD, McNicholas WT. Hypoxemia in patients with COPD: cause, effects, and disease progression. Int J Chron Obstruct Pulmon Dis 2011; 6: 199-208.

31 Sakao S, Voelkel NF, Tatsumi K. The vascular bed in COPD: pulmonary hypertension and pulmonary vascular alterations. Eur Respir Rev 2014; 23: 350-355.

32 Dournes G, Laurent F, Coste F, et al. Computed tomographic measurement of airway remodeling and emphysema in advanced chronic obstructive pulmonary disease. Correlation with pulmonary hypertension. Am J Respir Crit Care Med 2015; 191: 63-70.

33 Stone IS, Barnes NC, James WY, et al. Lung deflation and cardiovascular structure and function in chronic obstructive pulmonary disease. A randomized controlled trial. Am J Respir Crit Care Med 2016; 193: 717-726.

34 Bonderman D, Ghio S, Felix SB, et al. Riociguat for patients with pulmonary hypertension caused by systolic left ventricular dysfunction: a phase IIb double-blind, randomized, placebo-controlled, dose-ranging hemodynamic study. Circulation 2013; 128: 502-511.

35 Pyrgos G, Togias A, Brown RH. Phosphodiesterase V inhibition reduces airway responsiveness, but does not improve the beneficial effect of deep inspiration. Respiration 2013; 86: 243-251. 